\title{
Testicular Cancer - An Unexpected Course of the Disease
}

\author{
Antonia Mrdeža, ${ }^{1}$ Sanja Vušković, ${ }^{2}$ Marijana Ćorić, ${ }^{1,3}$ Milena Gnjidić, ${ }^{2}$ Marija Gamulin ${ }^{1,2 *}$ \\ ${ }^{1}$ University of Zagreb School of Medicine, Croatia \\ ${ }^{2}$ Department of Oncology, University Hospital Center Zagreb, Croatia \\ ${ }^{3}$ Clinical Department of Pathology and Cytology, University Hospital Center Zagreb, Croatia
}

\begin{abstract}
There are two major histologic types of testicular cancer: pure seminoma and nonseminomatous germ cell tumours which include embryonic carcinoma, choriocarcinoma, yolc sac tumours and teratomas. Rarely, in $2 \%$ of cases, teratomas may contain elements of somatic cancer, such as sarcoma or adenocarcinoma and it is then referred to as a "teratoma with somatic type malignancy". The histology of somatic malignant elements most commonly includes adenocarcinoma and various types of sarcomas; however, so far as the primitive neuroectodermal tumors (PNETs) are concerned the experience is quite limited. Here we report an unusual case of a testicular seminoma that relapsed 6 months after surgery as a teratoma with somatic neuroendocrine differentiation situated in retroperitoneal lymph nodes.
\end{abstract}

Keywords: Seminoma, Teratoma, Neuroectodermal tumour, Neuroendocrine differentiation

\begin{abstract}
Abbreviations: PNET: Primitive neuroectodermal tumour; GCT: Germ cell tumour; NET: Neuroendocrine tumour; PET: Positron emission Tomography; CT: Computed Tomography; PEB: Bleomycin, etoposide and cisplatin; PEI: Cisplatin, etoposide and ifosfamide; MDT: Multidisciplinary team; AFP: $\alpha$ - fetoprotein; HE: Hematoxylin and eosin
\end{abstract}

\section{Introduction}

Although testicular cancer is a rare tumour with an overall incidence of less than $1 \%$ annually, its incidence in developed countries among men aged 15 to 40 has risen during the past several decades. ${ }^{1,2}$ There are two major histologic types: pure seminoma and nonseminomatous germ cell tumours (non-GCT). ${ }^{3}$ The latter ones are rarer but also more aggressive and include embryonic carcinoma, choriocarcinoma, yolc sac tumours and teratomas. ${ }^{4}$ Among this wide morphological variety of different subtypes of germ cell malignancies, teratomas are the ones which can show a partial trans- formation into a malignant somatic-type tumour component. In the 2004 edition of the WHO Classification of Tumours, this entity was classified as "teratoma with somatic-type malignancy", which was defined as "teratoma containing a malignant component of a type typically encountered in other organs and tissues, e.g. sarcomas and carcinomas". ${ }^{5}$ Since teratoma is derived from all three germinal layers, an extensive variety of somatic malignancies have been described in the literature, but by far the most common are adenocarcinomas and sarcomas of any type ${ }^{6-8}$ On the other hand, testicular neuroendocrine tumour (NET) associated with teratoma is a distinct disease entity described in less than $2 \%$ of cases. ${ }^{9}$

Carcinoid tumours represent an interesting family of tumours that are derived from neuroendocrine cells. These tumours have diverse outcomes and may behave aggressively in some organs; therefore, some investigators have suggested that a designation of "neuroendocrine carcinoma" is more appropriate than "carci-

\begin{tabular}{|l|l|}
\hline \hline Quick Response Code: & *Corresponding author: Marija Gamulin, University of Zagreb School of Medicine, Šalata 3, \\
10000 Zagreb, Croatia & \multicolumn{1}{c}{ Published: 15 November, 2021} \\
Received: 20 September, 2021 & Citation: Mrdeža A, Vušković S, Corić M, Gnjidić M, Gamulin M. Testicular Cancer - An Unex- \\
pected Course of the Disease. SOJ Med Clin Case Rep. 2021;1(2):1-4. DOI: \\
$10.53902 / S O J M C C R .2021 .01 .000507$
\end{tabular}


noid tumor". ${ }^{10}$ Although teratoma coexists in a quarter of cases, ${ }^{11}$ neuroendocrine carcinoma can also be concomitantly present with seminoma in the same tumour but separately. ${ }^{12}$
We report a case of a metastatic retroperitoneal teratoma with transformation into somatic-type malignancy of neuroendocrine carcinoma that recurred 6 months after orchidectomy due to seminoma Figure 1.

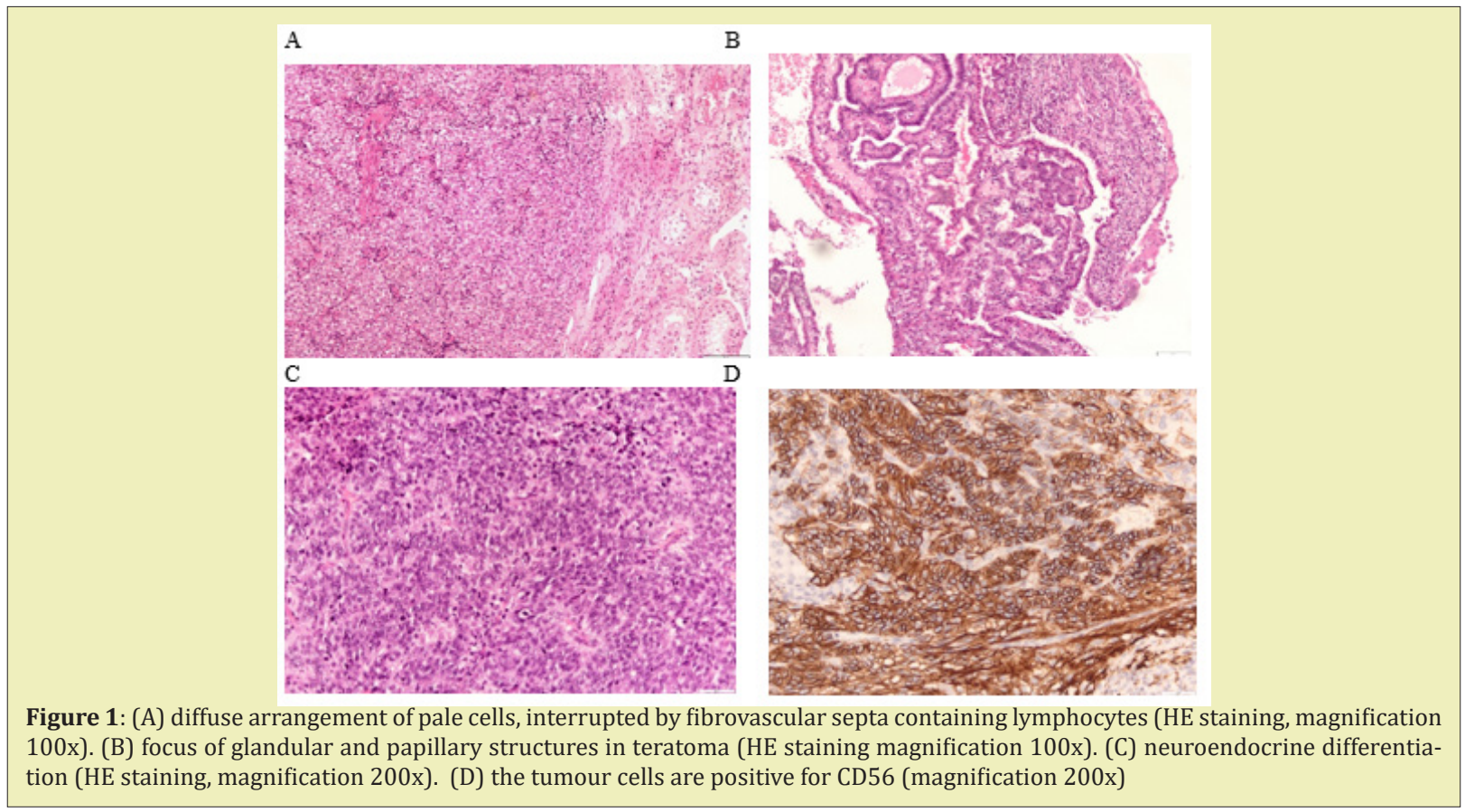

\section{Case Report}

A 25-year-old man with no previous medical history noticed painless swelling of his right testical in 4/2018. Clinical examination revealed a testicular cancer with biomarker levels $\beta$ HCG 12.9IU/L, AFP 63.8IJ/mL, LDH 190 U/L. Patient underwent a right-sided orchiectomy and according to pathologic report tumour was seminoma, pT2, stage IB. We requested revision of pathologic report which confirmed the original finding. Postoperatively, tumour biomarkers were $\beta$ HCG 0IU/L, AFP $5.5 \mathrm{IJ} / \mathrm{mL}$, LDH 180U/L. According to all diagnostic findings it was clear that we diagnosed seminoma with increased levels of AFP which is treated as nonseminomatous tumor.

Accordingly, we continued patient active surveillance until 12/2018 when PET-CT showed malignant activity in retroperitoneal lymph nodes measuring $5.3 \times 3.6 \mathrm{~cm}$. Due to the above, we decided to start a chemotherapy. Before starting chemotherapy, we encouraged the patient to do a semen cryopreservation. Also, from January to April of 2019 patient received a total of 3 cycles of PEB chemotherapy and radiologic surveillance reported regression of the disease. In 12/2019 PET-CT finding showed progression of retroperitoneal lymph nodes conglomerate. After MDT discussion, in 2/2020 an open biopsy and partial resection of the conglomerate was done. Pathologic report verified teratoma with somatic neuroendocrine differentiation reaching the resection margin. Soon after the biopsy, disease progressed, so therefore in 6/2020 resection of tumour conglomerate, Para aortal lymph nodes and right ureter was done. Pathologic report confirms teratoma with somatic neuroendocrine differentiation.

Two months after the surgery PET-CT showed disease progression with retroperitoneal nodules up to $2.4 \mathrm{~cm}$ in size and new pathologic neck lymph node. Patient presented with severe pain of the anal region, tenesmus and fever. Clinical examination showed the remaining testicle was tumour-free. Due to rapid disease progression in 9/2020 we started second-line PEI chemotherapy. Patient received a total of 6 cycles of chemotherapy so far with a delay of last cycle for a few days due to overcoming COVID 19 infection. After six cycles of therapy the patient is pain-free and PET-CT reports constantly show regression of the disease (Figures 2,3).

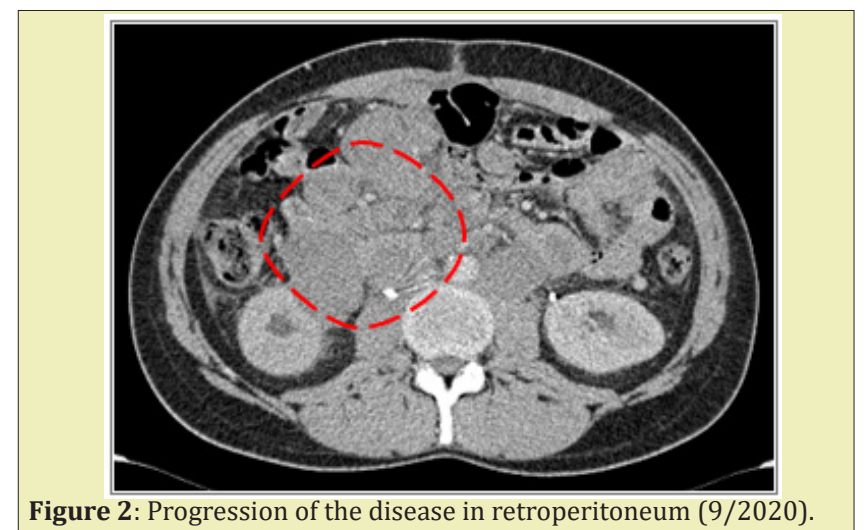




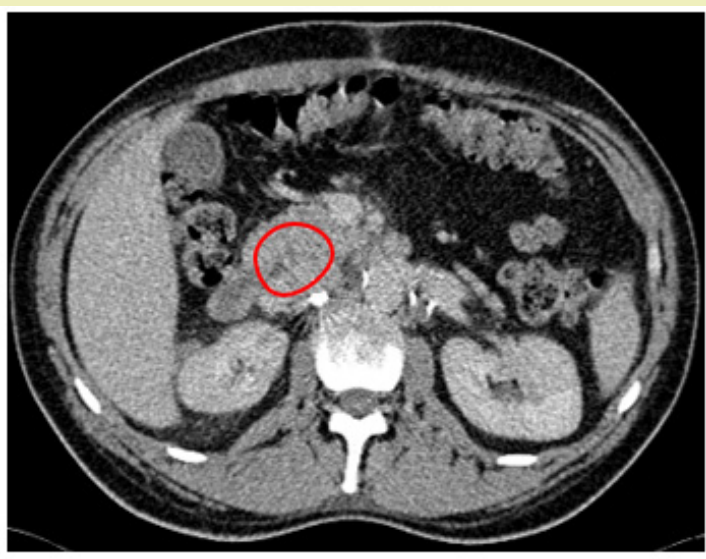

Figure 3: Regression of the disease after 6 cycles of PEI (1/2021)

\section{Discussion and Conclusion}

The main question in this case is whether this is teratoma with somatic type malignancy since two independent pathologic analysis reported seminoma after orchiectomy. There is documented instance of primary testicular NET coexisting with seminoma in an ipsilateral testis, ${ }^{13}$ but there are no recorded cases of a NET deriving from a pure seminoma. Also, it is important to mention that our patient had elevated levels of $\alpha$ - fetoprotein (AFP) before orchiectomy. AFP is a protein synthesised by the embryonal yolk sac and the liver of developing fetus and after birth is usually no longer detectable in the circulating blood stream. ${ }^{14}$ Pathologically, AFP is synthesised during hepatic diseases, and also by GCTs with components of yolk sac tumour. Although unspecific AFP elevations can occur in about $2 \%$ of seminoma patients, ${ }^{14}$ it is not secreted by pure seminoma in the absece of other sources of AFP elevation, such as liver dysfunction. Therefore, a histologic diagnosis of seminoma at orchiectomy with an elevation in serum AFP can indicate the presence of unrecognized nonseminomatous elements. ${ }^{3}$ There is a possibility that this is a case of an unrecognized non-seminoma component of the tumour due to inadequate tumour sampling. In addition, teratoma is often the only component that remains histologically detectable in residual lesions after platinum-based chemotherapy for metastatic mixed non-seminomatous GCT. ${ }^{15}$ There is a possibility that this is a case of an unrecognized non-seminoma component of the tumour due to inadequate tumour sampling.

Malignant transformation of germ cell tumours into somatic malignancy is not common but is associated with worse prognosis than classic germ cell tumors. ${ }^{7,16,17}$ General lack of response to traditional chemotherapeutic regimens aimed at GCT and locally aggressive nature of the disease contribute to poorer outcomes. ${ }^{18}$ Carcinoid tumour arising in a mature teratoma is a slow growing malignancy and there are usually no symptoms until the tumour was palpated or incidentally found. ${ }^{19}$ Therefore it is necessary to include neuroendocrine carcinoma in differential diagnosis of tes- ticular tumour with metastases. Also, somatic type malignancies have a dismal prognosis when found in metastatic sites rather than in testis and for that reason the correct diagnosis is very important to establish the therapy based on surgical approach. ${ }^{9}$

The origin of testicular neuroendocrine carcinoma is a matter of controversy and more studies are required to clarify the pathogenesis. Due to rarity of this cases, treatment is based on data obtained from several case reports and we need more reliable data from controlled clinical trials to increase treatment success (Figure 4).

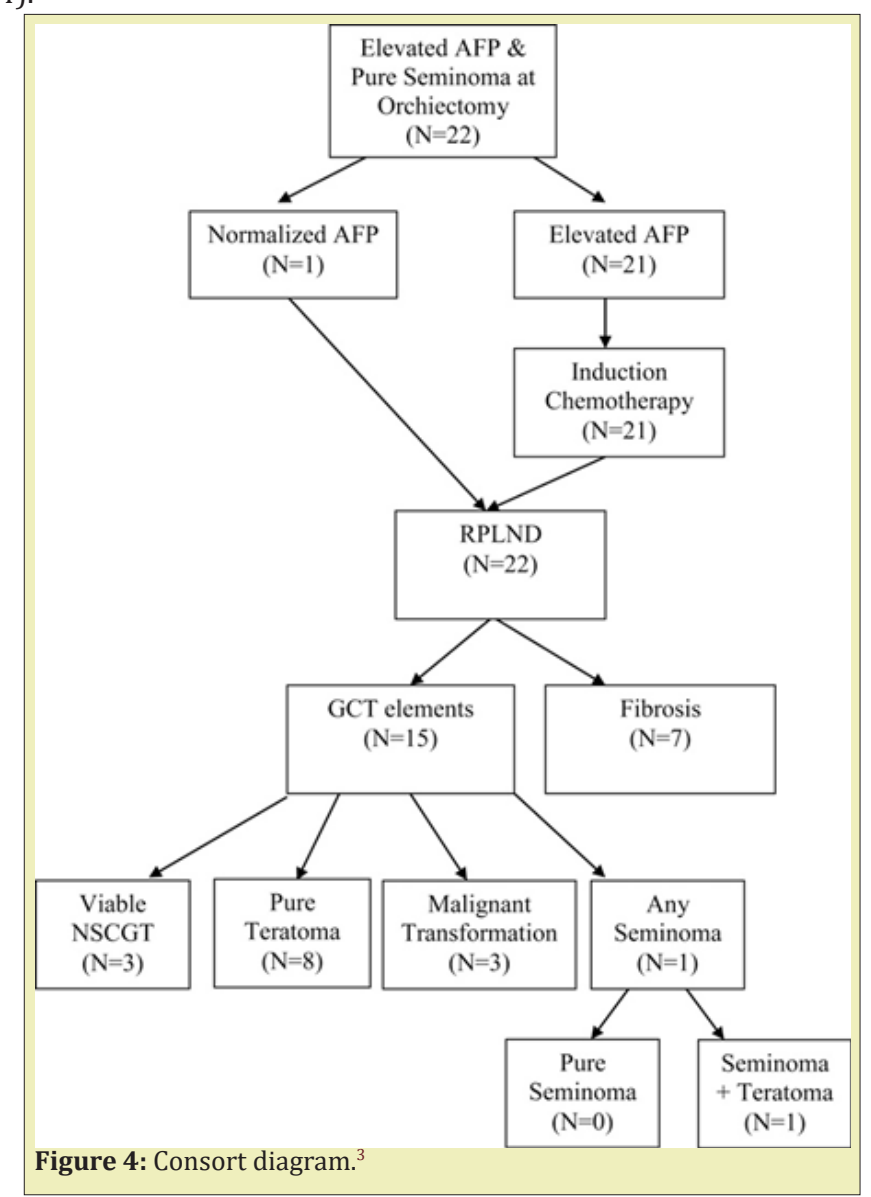

Acknowledgement

None.

Funding

None.

\section{Conflicts of Interest}

Author declares that there is no conflict of interest.

\section{References}

1. Shanmugalingam T Soultati A, Chowdhury S, et al. Global incidence and outcome of testicular cancer. Clin Epidemiol. 2013;5:417-427.

2. Purdue MP, Devesa SS, Sigurdson AJ, et al. International patterns and trends in testis cancer incidence. Int J Cancer. 2005;115:822-827. 
3. Kundu SD, Carver BS, Sheinfeld J. Retroperitoneal histologic findings of patients with elevated serum alpha-fetoprotein and pure seminoma at orchiectomy. Urology. 2011;8:844-847.

4. Gilligan TD, Hayes DF, Seidenfeld J, et al. American society of clinical oncology clinical practice guideline on uses of serum tumor markers in adult males with germ cell tumors. J Clin Oncol. 2010;28:3388-3404.

5. Chan TY. World Health Organization classification of tumours: Pathology \& genetics of tumours of the urinary system and male genital organs. Urology. 2005;65:214-215.

6. Motzer RJ, Amsterdam A, Prieto V, et al. Teratoma with malignant transformation: Diverse malignant histologies arising in men with germ cell tumors. J Urol. 1998;159:133-138.

7. Speir R, Cary C, Foster RS, et al. Management of patients with metastatic teratoma with malignant somatic transformation. Curr Opin Urol. 2018;28:469-473.

8. Mohanty SK, Balani JP, Parwani AV. Primitive Neuroectodermal Tumor Arising in a Testicular Teratoma with Retroperitoneal Metastasis: Report of an Interesting Case with Review of Literature. Urology. 2007;70:812.e7-812.e10.

9. Mikuz G, Colecchia M. Teratoma with somatic-type malignant components of the testis. A review and an update. Virchows Arch. 2012;461:27-32.

10. Reyes A, Moran CA, Suster S et al. Neuroendocrine Carcinomas (Carcinoid Tumor) of the Testis A Clinicopathologic and Immunohistochemical Study of Ten Cases. Am J Clin Pathol. 2003;120:182-187.

11. Thomas JC, Jones JS. Primary Carcinoid Tumor of the Testis Found at the Time of Elective Sterilization. Case Report. 2004;25:338-339.
12. Kumaki N, Umemura S, Kajiwara H, et al. Immunohistochemical Analysis of Neuroendocrine (NE) Differentiation in Testicular Germ Cell Tumors (GCTs): Use of Confocal Laser Scanning Microscopy (CLSM) to Demonstrate Direct NE Differentiation from GCTs. Acta Histochem Cytochem. 2007;40(6):143-151.

13. Iida N, Takemura K, Ito M, et al. Primary Testicular Neuroendocrine Tumor Coexisting With Seminoma Sharing Germ Cell Origin. Int J Surg Pathol. 2021;29(8):887-891.

14. Dieckmann KP, Anheuser P, Simonsen H, et al. Pure Testicular Seminoma with Non-Pathologic Elevation of Alpha Fetoprotein: A Case Series. Urol Int. 2017;99:353-357.

15. Zeh N, Wild PJ, Bode PK, et al. Retroperitoneal teratoma with somatic malignant transformation : A papillary renal cell carcinoma in a testicular germ cell tumour metastasis following platinum-based chemotherapy. BMC Urol. 2013;13:9.

16. Donadio BAC, Motzer RJ, Bajorin DF, et al. Chemotherapy for Teratoma with Malignant Transformation. J Clin Oncol. 2003;21:4285-4291.

17. Thai E, Leonardi F, Soliani P et al. Late Recurrence of a Seminoma of the Testis with a Poorly Differentiated Neuroendocrine Carcinoma Component. Int J Surg Pathol. 2012;20(4):396-400.

18. Ahmed T, Bosl GJ, Hajdu SI. Teratoma With Malignant Transformation in Germ Cell Tumors in Men. Cancer 1985;56:860-863.

19. Stroosma OB, Delaere KPJ. Carcinoid tumours of the testis. BJU Int. 2008;101:1101-1105. 